\title{
Pneumothorax rate in intubated patients with COVID-19
}

\author{
Kathleen M. Capaccione ${ }^{1}$, Belinda D'souza' ${ }^{1}$, Jay Leb ${ }^{1}$, Lyndon Luk ${ }^{1}$, Jimmy Duong ${ }^{2}$, Wei-Yann Tsai , \\ Ben Navot ${ }^{1}$, Shifali Dumeer ${ }^{1}$, Ahmed Mohammed $^{3}$, Mary M. Salvatore ${ }^{1}$ \\ ${ }^{1}$ Division of Cardiothoracic Imaging, Department of Radiology, Columbia University Irving Medical Center, New York, NY; ${ }^{2}$ Department of Statistics, Mailman \\ School of Public Health, Columbia University, New York, NY; ${ }^{3}$ Division of Critical Care, Department of Medicine, The Mount Sinai Hospital, New York, NY, USA
}

\section{Dear Editor:}

Coronavirus disease 2019 (COVID-19) has emerged as a major public health crisis. Initial presentation includes headache, fever, cough, and myalgia however many patients develop severe systemic illness requiring critical care [1]. Patients with COVID-19 have a high incidence of pulmonary barotrauma (PBT) [2]. We investigated the rate of pneumothorax in critically ill intubated patients with COVID-19 infection.

Under our HIPAA-compliant IRB-approved protocol (approved by the Columbia University Human Research Protection Office and Institutional Review Board, protocol number AAAS9652 approved March 16, 2020), we retrospectively queried our hospital's medical record system for chest radiograph reports containing "endotracheal tube", "ETT", or "intubated" and "COVID-19" between February 12, 2020 and April 8, 2020. Given the uncertainty of COVID-19 infection status on admission, this better captured COVID-19 status than International Classification of Diseases, tenth revision (ICD-10) codes at the time of this study. Informed consent was not obtained given the retrospective nature of this study. All patients included in analysis were intubated during their hospitalization. We performed retrospective chart review to obtain information on demographics, COVID-19 infection status, ventilator settings, and duration of intubation and outcome. Finalized radiograph reports were used to identify if a pneumothorax was present; each radiograph had been read for clinical use by a single attending radiologist, with experience ranging from 2 to 27 years.

Studies from 221 unique patients were identified; the patient selection process is shown in Figure 1. Studies performed at an affiliate community hospital were excluded, resulting in 198 unique patients. The first 150 by sequential medical record numbers were analyzed. Eighteen patients who had missing data or who were ultimately found to be COVID-19 negative were excluded, resulting in a total of 132 unique patients. For statistical analysis, Student ttests and chi-square tests were used for continuous and categorical variables, respectively, to evaluate differences between patients with and without pneumothorax. A Cox proportionalhazards model was performed to assess the hazard ratio of pneumothorax as a result of intubation time. We adjusted for covariates of tidal volume (TV) and positive end expiratory pressure (PEEP).

Of the 132 patients studied, average age was 63 years (range, 25-90 years); 83 of 132 were male (62\%), 18 had pneumothorax (14\%), and 61 had died at 4 months after the data accrual

\section{Letter to the Editor}

Received: September 1, 2020

Revised: November 26, 2020

Accepted: December 1, 2020

Corresponding author Kathleen M. Capaccione Department of Radiology, Columbia University Irving Medical Center, 622 W 168th St, New York, NY 10032, USA Tel: +1-212-305-9024

Fax: +1-212-305-5777

E-mail: kmc9020@nyp.org

Copyright (@) 2021 The Korean Society of Critical Care Medicine

This is an Open Access article distributed under the terms of Creative Attributions Non-Commercial License (https:// creativecommons.org/li-censes/by-nc/4.0/) which permits unrestricted noncommercial use, distribution, and reproduction in any medium, provided the original work is properly cited. 


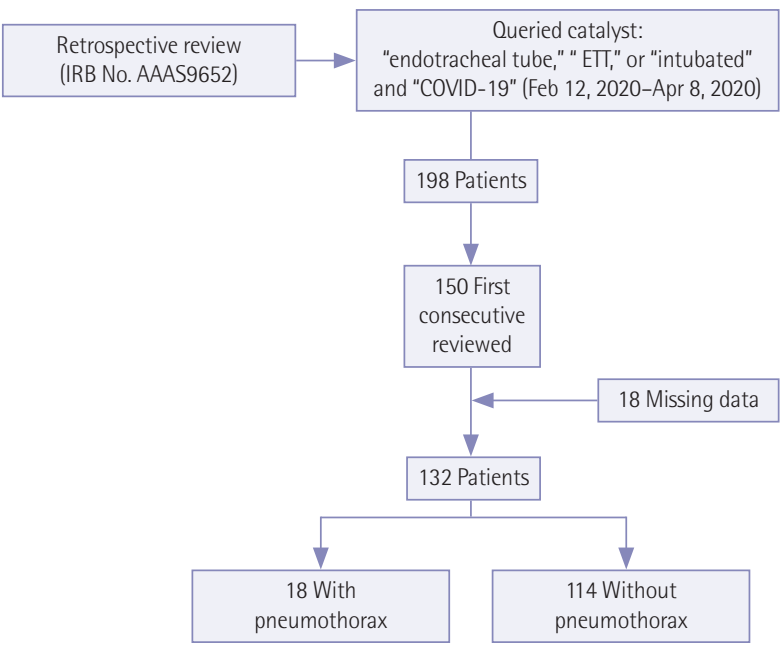

Figure 1. Selection criteria for patients included in this study. IRB: Institutional Review Board; COVID-19: coronavirus disease 2019.

Table 1. Demographic and clinical data for all patients studied

\begin{tabular}{lc}
\hline Variable & Value \\
\hline Number of patients & 132 \\
Age $(\mathrm{yr})$ & $63(25-90)$ \\
Male sex & $83(62)$ \\
Pneumothorax & $18(14)$ \\
Death rate & $61(46)$ \\
Average initial respiratory rate (breaths $/ \mathrm{min})$ & 24.8 \\
Average initial PEEP (cm $\left.\mathrm{H}_{2} \mathrm{O}\right)$ & 13.1 \\
Average initial tidal volume $(\mathrm{mL})$ & 394.9 \\
Average initial FiO $2 \%)$ & 72.0 \\
\hline
\end{tabular}

Values are presented as median (range) or number (\%). Death rate was calculated at 4 months post-completion of the study accrual period.

PEEP: positive end expiratory pressure; $\mathrm{FiO}_{2}$ : fraction of inspired oxygen.

period was completed (46\%) (Table 1). Ventilator settings including respiratory rate, PEEP, TV, and fraction of inspired oxygen $\left(\mathrm{FiO}_{2}\right)$ are also described in Table 1. Figure 2 demonstrates pneumothoraces in patients infected with COVID-19. Moderate-to-large pneumothoraces were treated with chest tube insertion, while small pneumothoraces were observed with serial radiography to resolution. In 16 of 18 patients (89\%), chronicity data was available and in all cases pneumothorax occurred after intubation. Of these 16, pneumothorax occurred on the day of intubation in three cases; in the remaining 13 the average time after intubation when pneumothorax developed was 14.9 days (standard deviation [SD], 10.0). Six of the 16 patients died with a pneumothorax; of the 10 that survived the average time to resolution of pneumothorax was 2.7 days (SD, 2.4).

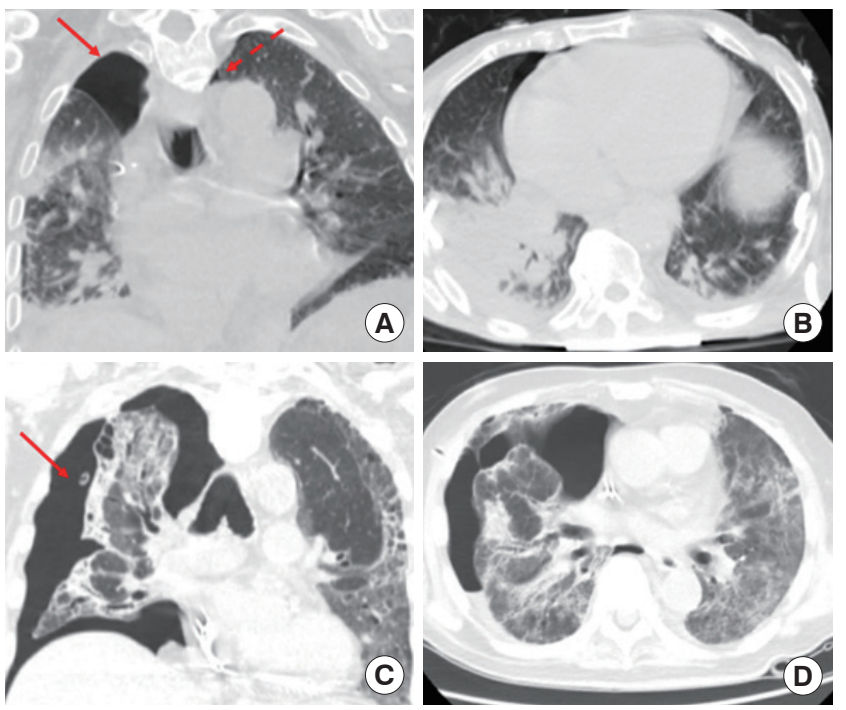

Figure 2. Coronavirus disease 2019 (COVID-19)-positive patients with small (dashed arrow) and moderate pneumothoraces (solid arrow) (A, B), and large pneumothorax (solid arrow) (C, D) in the coronal and axial planes.

Table 2. Demographic and clinical characteristics of patients with and without pneumothorax

\begin{tabular}{lccc}
\hline Variable & Pneumothorax & No pneumothorax & P-value \\
\hline No. of patients & 18 & 114 & - \\
Age (yr), median (range) & $57(25-80)$ & $64(32-90)$ & 0.04 \\
Male sex (\%) & 15 & 68 & 0.09 \\
$\begin{array}{l}\text { Average initial PEEP } \\
\left.\text { (cm } \mathrm{H}_{2} \mathrm{O}\right)\end{array}$ & 12.1 & 13.3 & 0.31 \\
$\begin{array}{l}\text { Average initial tidal } \\
\text { volume (mL) }\end{array}$ & 405.7 & 393.1 & 0.43 \\
$\begin{array}{l}\text { Average initial respiratory } \\
\text { rate (breaths/min) }\end{array}$ & 24.9 & 24.8 & 0.97 \\
\begin{tabular}{l} 
Average initial $\mathrm{FiO}_{2}(\%)$ \\
\hline
\end{tabular} & 90.6 & 69.0 & $<0.001$ \\
\hline
\end{tabular}

Patients with pneumothorax were significantly younger than those without $(P=0.04)$. Average $\mathrm{FiO}_{2}$ was higher in patients with pneumothorax than those without $(\mathrm{P}<0.001)$.

PEEP: positive end expiratory pressure; $\mathrm{FiO}_{2}$ : fraction of inspired oxygen.

Of the patients with and without pneumothorax, patients with pneumothorax had an average age of 57 (SD, 15.0) compared to those without pneumothorax with an average of 64 (SD, 12.4; $\mathrm{P}=0.04$ ). Table 2 summarizes demographic and clinical characteristics as well as initial ventilator settings of patients with and without pneumothorax. Given the importance of ventilator settings in PBT, we analyzed initial ventilator settings for patients with and without pneumothorax. Initial ventilator settings were available for 103 of 114 patients without pneumothorax and 17 of 18 with pneumothorax. Patients with pneumothorax had statistically significantly higher 
average initial $\mathrm{FiO}_{2}(\mathrm{P}<0.001)$. There was no difference in average PEEP between the groups, with values of 12.1 and 13.3 $\mathrm{cm}_{2} \mathrm{O}$ for patients with and without pneumothorax, respectively $(\mathrm{P}=0.31)$. Average TV were $405.7 \mathrm{~mL}$ and $393.1 \mathrm{~mL}$ for patients with and without pneumothorax respectively $(\mathrm{P}=0.40)$. To evaluate the effects intubation time on the rate of pneumothorax, we performed a Cox regression model analyzing pneumothorax as the time-dependent covariate. Without adjusting for additional covariates, the hazard ratio of pneumothorax was $1.99(\mathrm{P}=0.08)$. Adjusting for the covariates of PEEP and $\mathrm{TV}$, the hazard ratio of pneumothorax was $1.71(\mathrm{P}=0.21)$, hazard ratio of TV was $1.00(\mathrm{P}=0.11)$ and hazard ratio of PEEP was 1.03 $(\mathrm{P}=0.36)$.

Clinical severity scores were not available; however we assigned a grade to each pneumothorax from 1 being least severe and 4 being most severe; average grade was 2.1. There was no correlation with pneumothorax grade and length or intubation, hospitalization, or survival. We sought to identify if body mass index (BMI) or smoking history correlated with the incidence of pneumothorax, but found that the majority of patients were lacking data on BMI ( $89 \%$ and $83 \%$ for patients without and with pneumothorax, respectively), and were lacking data on smoking history ( $52 \%$ and $83 \%$ for patients without and with pneumothorax, respectively).

Pulmonary disease predisposes to pneumothorax; we therefore assessed if patients with pneumothorax had a higher rate of underlying pulmonary disease. None of the 18 patients with pneumothorax had chronic obstructive pulmonary disease (COPD), pulmonary fibrosis, asthma, lung cancer, or pulmonary artery hypertension (PAH). Of patients without pneumothorax, four had COPD (4\%) and five had asthma (4\%); none had pulmonary fibrosis, lung cancer, or PAH.

Here, we demonstrate a $14 \%$ pneumothorax rate in COVID-19 positive intubated critically ill patients, significantly higher than the $2.9 \%$ in mechanically ventilated in the intensive care unit (ICU) reported by Anzueto et al. [3]. They demonstrated differences in rates of barotrauma based on underlying pulmonary pathology, however this was not observed in our study, although the incidence of underlying pulmonary disease was low. Previous studies have shown that incidence of PBT increases over time [4]. Average length of intubation in our study was much longer than prior studies, which may in part account for the high rate of PBT. COVID-19 infection has been established as distinct from other types of acute respiratory distress syndrome (ARDS) by its presentation with severe hypoxemia in the setting of near-normal respiratory compliance. The distinct effects of COVID-19 on the lung may com- promise structural integrity and predispose to PBT.

Clinically, the risk of barotrauma must be balanced with potential improved alveolar recruitment in the setting of high PEEP. In our study, there was no difference in the average PEEP in patents with or without pneumothorax. A meta-analysis with 3,612 patients by Guo et al. [5], demonstrated that patients who responded to PEEP by increased oxygenation had reduced hospital mortality, ICU mortality, and 28-day mortality. Importantly, they found no significant difference in the risk of PBT in patients receiving high versus low PEEP, consistent with our analysis [5]. As further investigation yields a better understanding of pulmonary changes resulting from COVID-19, ventilator strategies will continue to be refined to optimize the risk-benefit ratio for patients.

Limitations were that we only included a single center with extensive critical care experience given that we serve as major tertiary referral center for complex critically ill patients in our region, treating over 1,300 critically ill patients yearly, limiting generalizability of results. We compared the rate of pneumothorax in COVID-19 positive patients to published rates in the literature; an ideal group for comparison would have been intubated critically ill COVID-19 negative patients during the same time period; however there were not enough patients meeting these criteria for a valid statistical comparison. Our query yielded five critically ill intubated patients who tested negative for COVID-19 and one patient who was never tested.

Despite these limitations, our data demonstrate a high rate of pneumothorax in COVID-19 positive patients, with no effect of ventilator settings of PEEP or TV and tidal volume on the rate of pneumothorax. Given prior studies demonstrating that underlying pulmonary disease predisposes to PBT, we evaluated underlying pulmonary disease; however, there was no difference in rate of pneumothorax based on underlying pulmonary disease.

These data are concordant with other groups that have established that COVID-19 pulmonary disease is distinct from previously described ARDS. The long intubation in COVID-19 patients may contribute to the high rate of PBT; here we show that ventilator setting may not contribute significantly to outcomes. Future study will further characterize the phenotypes of COVID-19 ARDS and help clinicians optimize ventilation strategies for patients with COVID-19.

\section{CONFLICT OF INTEREST}

No potential conflict of interest relevant to this article was reported. 


\section{ORCID}

Kathleen M.Capaccione https://orcid.org/0000-0002-6536-2673

Jay Leb

Lyndon Luk https://orcid.org/0000-0002-9940-2610

Shifali Dumeer https://orcid.org/0000-0002-4468-3128

Mary M. Salvatore https://orcid.org/0000-0003-1950-7795 https://orcid.org/0000-0002-1429-5749

\section{AUTHOR CONTRIBUTIONS}

Conceptualization: KMC, BD, MMS. Data curation: KMC, BD, LL, JL, BN. Formal analysis: KMC, JD, WT. Methodology, Project administration, \& Visualization: KMC, BD, MMS. Writingoriginal draft: KMC. Writing-review \& editing: BD, LL, JL, LL, BN, SD, JD, AM, WT.

\section{REFERENCES}

1. Grasselli G, Pesenti A, Cecconi M. Critical care utilization for the COVID-19 outbreak in Lombardy, Italy: early experience and forecast during an emergency response. JAMA 2020;323: 1545-6.

2. McGuinness G, Zhan C, Rosenberg N, Azour L, Wickstrom M, Mason DM, et al. Increased incidence of barotrauma in patients with COVID-19 on invasive mechanical ventilation. Radiology 2020;297:E252-62.

3. Anzueto A, Frutos-Vivar F, Esteban A, Alía I, Brochard L, Stewart $\mathrm{T}$, et al. Incidence, risk factors and outcome of barotrauma in mechanically ventilated patients. Intensive Care Med 2004;30:612-9.

4. Gattinoni L, Bombino M, Pelosi P, Lissoni A, Pesenti A, Fumagalli $\mathrm{R}$, et al. Lung structure and function in different stages of severe adult respiratory distress syndrome. JAMA 1994; 271:1772-9.

5. Guo L, Xie J, Huang Y, Pan C, Yang Y, Qiu H, et al. Higher PEEP improves outcomes in ARDS patients with clinically objective positive oxygenation response to PEEP: a systematic review and meta-analysis. BMC Anesthesiol 2018;18:172. 